Journal of Engineering and Applied Sciences 14 (Special Issue 7): 9966-9972, 2019

ISSN: 1816-949X

(C) Medwell Journals, 2019

\title{
Subclass for Higher-Order Derivatives of Multivalent Analytic Functions on Complex Hilbert Space with Some Applications in Fractional Calculus
}

\author{
${ }^{1}$ Karrar Khudhair Obayes, ${ }^{2}$ Rasha Abbas Isewid and ${ }^{3}$ Abbas Kareem Wanas \\ ${ }^{1}$ Department of Mathematics, College of Computer Science and Information Technology, \\ ${ }^{2}$ College of Physical Education and Sport Science, \\ ${ }^{3}$ College of Computer Science and Information Technology, University of Al-Qadisiyah, \\ Al-Diwaniyah, Iraq
}

\begin{abstract}
In the present investigation, we introduce and study a certain subclass for higher-order derivatives of multivalent analytic functions defined on complex Hilbert space. We determine some properties of this class, like, coefficient estimates, radii of starlikeness and convexity and convex combination. Also, we give an applications of the fractional calculus techniques.
\end{abstract}

Key words: Multivalent functions, higher-order derivatives, Hilbert space, radii of starlikeness and convexity, fractional calculus, coefficient

\section{INTRODUCTION}

Let $A_{p}^{m}$ indicate the family of all functions $f$ of the form:

$$
\begin{aligned}
& \mathrm{f}(\mathrm{z})=\mathrm{z}^{\mathrm{p}}+\sum_{\mathrm{n}=\mathrm{m}}^{\infty} \mathrm{a}_{\mathrm{n}} \mathrm{z}^{\mathrm{n}} \\
& (\mathrm{p}<\mathrm{m}, \mathrm{p}, \mathrm{m} \in \mathrm{N}=\{1,2, \ldots\})
\end{aligned}
$$

Which are analytic and multivalent in the open unit disk $U=\{z \in C:|z|<1\}$. Let $K_{p}^{m}$ denote the subclass of $A_{p}^{m}$ consisting of functions of the form:

$$
\begin{aligned}
& f(z)=z^{p}-\sum_{n=m}^{\infty} a_{n} z^{n} \\
& \left(a_{n} \geq 0, p<m, p, m \in N=\{1,2, \ldots\}\right)
\end{aligned}
$$

Upon differentiating both sides of (1.2) $\alpha$ times with respect to $\mathrm{z}$, we obtain (Chen et al., 1995):

$$
\begin{aligned}
& \mathrm{f}^{(\alpha)}(\mathrm{z})=\varphi(\mathrm{p}, \alpha) \mathrm{z}^{\mathrm{p}-\alpha}+\sum_{\mathrm{n}=\mathrm{m}}^{\infty} \varphi(\mathrm{n}, \alpha) \mathrm{a}_{\mathrm{n}} \mathrm{z}^{\mathrm{n}-\alpha} \\
& \left(\mathrm{p}, \mathrm{m} \in \mathrm{N} ; \alpha \in \mathrm{N}_{0}=\mathrm{N} \cup\{0\} ; \mathrm{p}>\alpha\right)
\end{aligned}
$$

Where:

$$
\begin{aligned}
& \varphi(\mathrm{p}, \alpha)=\frac{\mathrm{p} !}{(\mathrm{p}-\alpha) !}= \\
& \left\{\begin{array}{cc}
1 & (\alpha=0) \\
\mathrm{p}(\mathrm{p}-1) \cdots(\mathrm{p}-\alpha+1) & (\alpha \neq 0)
\end{array}\right.
\end{aligned}
$$

Several researchers have investigated higher-order derivatives of multivalent functions, see for example (Altıntas, 2007; Irmak and Cho, 2007; Wanas, 2015; Wanas, 2017 and Wanas and Majeed, 2018). A function $\mathrm{f} \in \mathrm{A}_{\mathrm{p}}^{\mathrm{m}}$ is said to be multivalent starlike of order $\gamma(0 \leq \gamma<p)$ ifit satisfies the condition:

$$
\operatorname{Re}\left\{\frac{\mathrm{zf}^{\prime}(\mathrm{z})}{\mathrm{f}(\mathrm{z})}\right\}>\gamma(\mathrm{z} \in \mathrm{U})
$$

And is said to be multivalent convex of order $\gamma$ $(0 \leq \gamma<p)$ if it satisfies the condition:

$$
\operatorname{Re}\left\{1+\frac{\mathrm{zf}^{\prime \prime}(\mathrm{z})}{\mathrm{f}^{\prime}(\mathrm{z})}\right\}>\gamma(\mathrm{z} \in \mathrm{U})
$$

Denote by $S_{m}^{*}(p, \gamma)$ and $C_{m}(p, \gamma)$ the classes of multivalent starlike and multivalent convex functions of $\gamma$ order, respectively which were introduced by Owa (1992). It is known that (Goodman, 1983) and (Owa, 1985): 


$$
\mathrm{f} \in \mathrm{C}_{\mathrm{m}}(\mathrm{p}, \gamma) \text { if andonlyif } \frac{\mathrm{zf}^{\prime}(\mathrm{z})}{\mathrm{p}} \in \mathrm{S}_{\mathrm{m}}^{*}(\mathrm{p}, \gamma)
$$

The classes $\mathrm{S}_{\mathrm{m}}^{*}=\mathrm{S}^{*}(\mathrm{p}, \gamma)$ and $\mathrm{C}_{1}(\mathrm{p}, \gamma)=\mathrm{C}(\mathrm{p}, \gamma)$ were studied by Owa (1985). Let $\mathrm{H}$ be a complex Hilbert space and $\mathrm{T}$ be a bounded linear operator on $\mathrm{H}$. For a complex analytic function $\mathrm{f}$ on the Unit disk $\mathrm{U}$, we denoted $\mathrm{f}(\mathrm{T})$, the operator on $\mathrm{H}$ defined by the usual Riesz-Dunford integral (Dunford and Schwarz, 1988):

$$
f(T)=\frac{1}{2 \pi \mathrm{i}} \int_{C} f(z)(z I-T)^{-1} d z
$$

Where:

$\mathrm{I}=$ The Identity operator on $\mathrm{H}, \mathrm{C}$ is a positively oriented simple closed rectifiable contour lying in

$\mathrm{U}=$ Containing the spectrum $\sigma(\mathrm{T})$

$\mathrm{T}=$ Interior domain (Fan, 1978)

Also $f(T)$ can be defined by the following series:

$$
f(T)=\sum_{n=0}^{\infty} \frac{f^{(n)}(0)}{n !} T^{n}
$$

Which converges in the norm topology (Fan, 1979).

Definition 1.1 (Selvaraj et al., 2009): The fractional integral operator of order $\lambda(\lambda>0)$ is defined by:

$$
\mathrm{D}_{\mathrm{T}}^{-\lambda} \mathrm{f}(\mathrm{T})=\frac{1}{\Gamma(\lambda)} \int_{0}^{1} \frac{\mathrm{T}^{\lambda} \mathrm{f}(\mathrm{tT})}{(1+\mathrm{t})^{1-\lambda}} \mathrm{dt}
$$

where, $\mathrm{f}$ is analytic function in a simple connected region of z-plane containing the origin.

Definition 1.2 (Selvaraj et al., 2009): The fractional derivative for operator of order $\lambda(0 \leq \lambda<1)$ is defined by:

$$
D_{T}^{\lambda} f(T)=\frac{1}{\Gamma(1-\lambda)} \frac{d}{d T} \int_{0}^{1} \frac{T^{1-\lambda} f(t T)}{(1-t)^{\lambda}} d t
$$

where, $\mathrm{f}$ is analytic in a simply connected region of the z-plane containing the origin. For $f \in \mathrm{K}_{\mathrm{p}}^{\mathrm{m}}$, from definitions 1.1 and 1.2 by applying a simple calculation, we get:

$$
\mathrm{D}_{\mathrm{T}}^{-\lambda} \mathrm{f}(\mathrm{T})=\frac{\Gamma(\mathrm{p}+1)}{\Gamma(\mathrm{p}+\lambda+1)} \mathrm{T}^{\mathrm{p}+\lambda}-\sum_{\mathrm{n}=\mathrm{m}}^{\infty} \frac{\Gamma(\mathrm{n}+1)}{\Gamma(\mathrm{n}+\lambda+1)} \mathrm{a}_{\mathrm{n}} \mathrm{T}^{\mathrm{n}+\lambda}
$$

And:

$$
\begin{aligned}
& D_{T}^{\lambda} f(T)=\frac{\Gamma(p+1)}{\Gamma(p-\lambda+1)} T^{p-\lambda}- \\
& \sum_{n=m}^{\infty} \frac{\Gamma(n+1)}{\Gamma(n-\lambda+1)} a_{n} T^{n-\lambda}
\end{aligned}
$$

Definition 1.3: A function $f \in K_{p}^{m}$ is said to be in the class $\mathrm{AK}_{\mathrm{p}}^{\mathrm{m}}(\alpha, \beta, \delta, \mathrm{T})$ if satisfies the inequality:

$$
\left\|T^{\alpha} f^{(\alpha+\beta)}(T)-\frac{(p-\beta) !}{(p-\alpha-\beta) !} f^{(\beta)}(T)\right\|<\left\|\delta f^{(\beta)}(T)\right\|
$$

where, $\mathrm{p} \in \mathrm{N}, \alpha, \beta \in \mathrm{N}_{0}=\mathrm{N} \cup\{0\}, \alpha+\beta<\mathrm{p}, \delta \in \mathrm{C} \backslash\{0\} \backslash$ and for all operator $\mathrm{T}$ with $\|\mathrm{T}\|<1, \mathrm{~T} \neq \varnothing$ (Ø denote the zero operator on $\mathrm{H}$ ).

\section{MATERIALS AND METHODS}

Coefficient estimates: In this study, we derive coefficient estimates for the function $f$ to be in the class $\mathrm{AK}_{\mathrm{p}}^{\mathrm{m}}(\alpha, \beta, \delta, \mathrm{T})$.

Theorem 2.1: Let $f \in K_{p}^{m}$ be defined by (1.2). Then $\mathrm{f} \in \mathrm{AK}_{\mathrm{p}}^{\mathrm{m}}(\alpha, \beta, \delta, \mathrm{T})$ for all $\mathrm{T} \neq \varnothing$ if and only if:

$$
\begin{aligned}
& \sum_{n=m}^{\infty} \frac{n !}{(n-\beta) !}\left[\frac{(n-\beta) !}{(n-\alpha-\beta) !}-\frac{(p-\beta) !}{(p-\alpha-\beta) !}+|\delta|\right] a_{n} \leq|\delta| \\
& \frac{p !}{(p-\beta) !}
\end{aligned}
$$

where, $p \in N, \alpha, \beta \in N_{0}=N \cup\{0\}, \alpha+\beta<p, \delta \in C \backslash\{0\}$. The result is sharp for the function $\mathrm{f}$ given by:

$$
f(z)=z^{p}-\frac{|\delta| p !(n-\beta) !}{n !(p-\beta) !\left[\begin{array}{l}
\frac{(\mathrm{n}-\beta) !}{(\mathrm{n}-\alpha-\beta) !} \\
\frac{(\mathrm{p}-\beta) !}{(\mathrm{p}-\alpha-\beta) !}+|\delta|
\end{array}\right]} \mathrm{z}^{\mathrm{n}},(\mathrm{n} \geq \mathrm{m})
$$

Proof: Assume that the inequality (2.1) holds. Then, we get: 


$$
\begin{aligned}
& \left\|T^{\alpha} f^{(\alpha+\beta)}(T)-\frac{(p-\beta) !}{(p-\alpha-\beta) !} f^{(\beta)}(T)\right\|-\left\|\delta f^{(\beta)}(T)\right\|= \\
& \left\|\sum_{n=m}^{\infty} \frac{n !}{(n-\beta) !}\left[\frac{(n-\beta) !}{(n-\alpha-\beta) !}-\frac{(p-\beta) !}{(p-\alpha-\beta) !}\right] a_{n} T^{n-\beta}\right\|-\left\|\delta \frac{p !}{(p-\beta) !} T^{p-\beta}-\sum_{n=m}^{\infty} \delta \frac{n !}{(n-\beta) !} a_{n} T^{n-\beta}\right\| \\
& \leq \sum_{n=m}^{\infty} \frac{n !}{(n-\beta) !}\left[\frac{(n-\beta) !}{(n-\alpha-\beta) !}-\frac{(p-\beta) !}{(p-\alpha-\beta) !}\right] a_{n}\|T\|^{n-\beta}-|\delta| \frac{p !}{(p-\beta) !}\|T\|^{p-\beta}+|\delta| \sum_{n=m}^{\infty} \frac{n !}{(n-\beta) !} a_{n}\|T\|^{n-\beta} \\
& \leq \sum_{n=m}^{\infty} \frac{n !}{(n-\beta) !}\left[\frac{(n-\beta) !}{(n-\alpha-\beta) !}-\frac{(p-\beta) !}{(p-\alpha-\beta) !}+|\delta|\right] a_{n}-|\delta| \frac{p !}{(p-\beta) !} \leq 0
\end{aligned}
$$

Therefore, $f \in \mathrm{AK}_{\mathrm{p}}^{\mathrm{m}}(\alpha, \beta, \delta, \mathrm{T})$. To show the converse, let $\mathrm{f} \in \mathrm{AK}_{\mathrm{p}}^{\mathrm{m}}(\alpha, \beta, \delta, \mathrm{T})$. Then:

$$
\left\|T^{\alpha} f^{(\alpha+\beta)}(T)-\frac{(p-\beta) !}{(p-\alpha-\beta) !} f^{(\beta)}(T)\right\|<\left\|\delta f^{(\beta)}(T)\right\|
$$

Simple calculations gives:

$$
\begin{aligned}
& \left\|\sum_{n=m}^{\infty} \frac{n !}{(n-\beta) !}\left[\frac{(n-\beta) !}{(n-\alpha-\beta) !}-\frac{(p-\beta) !}{(p-\alpha-\beta) !}\right] a_{n} T^{n-\beta}\right\|< \\
& \left\|\delta \frac{p !}{(p-\beta) !} T^{p-\beta}-\sum_{n=m}^{\infty} \delta \frac{n !}{(n-\beta) !} a_{n} T^{n-\beta}\right\|
\end{aligned}
$$

Taking $\mathrm{T}=\gamma \mathrm{l}(0<\gamma<1)$ in the above inequality, we have:

$$
\frac{\sum_{n=m}^{\infty} \frac{n !}{(n-\beta) !}\left[\frac{(n-\beta) !}{(n-\alpha-\beta) !}-\frac{(p-\beta) !}{(p-\alpha-\beta) !}\right] a_{n} \gamma^{n-\beta}}{|\delta| \frac{p !}{(p-\beta) !} \gamma^{p-\beta}-\sum_{n=m}^{\infty}|\delta| \frac{n !}{(n-\beta) !} a_{n} \gamma^{n-\beta}}<1
$$

Upon clearing denominator in (2.3) and letting $\gamma \rightarrow 1$, we obtain:

$$
\begin{aligned}
& \sum_{n=m}^{\infty} \frac{n !}{(n-\beta) !}\left[\frac{(n-\beta) !}{(n-\alpha-\beta) !}-\frac{(p-\beta) !}{(p-\alpha-\beta) !}\right] \\
& a_{n}<|\delta| \frac{p !}{(p-\beta) !}-\sum_{n=m}^{\infty}|\delta| \frac{n !}{(n-\beta) !} a_{n}
\end{aligned}
$$

Or:
This completes the proof of the theorem.

Corollary 2.1: If $\mathrm{f} \in \mathrm{AK}_{\mathrm{p}}^{\mathrm{m}}(\alpha, \beta, \delta, \mathrm{T})$ then:

$$
a_{n} \leq \frac{|\delta| p !(n-\beta) !}{n !(p-\beta) !\left[\frac{(n-\beta) !}{(n-\alpha-\beta) !}-\frac{(p-\beta) !}{(p-\alpha-\beta) !}+|\delta|\right]},(n \geq m)
$$

\section{Radii of starlikeness and convexity}

Theorem 3.1: If $\mathrm{f} \in \mathrm{AK}_{\mathrm{p}}^{\mathrm{m}}(\alpha, \beta, \delta, \mathrm{T})$ then $\mathrm{f}$ will be p-valently starlike of order $\gamma(0 \leq \gamma<p)$ in the disk $|z|<\gamma_{1}$ where:

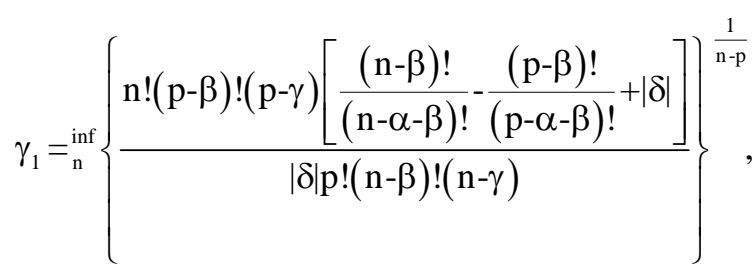

$$
(\mathrm{n} \geq \mathrm{m})
$$

The result is sharp for the function $\mathrm{f}$ given by (2.2).

Proof: It is enough to show that:

$$
\left\|\frac{T f^{\prime}(T)}{f(T)}-p\right\| \leq p-\gamma
$$

We have: 


$$
\left\|\frac{T f^{\prime}(T)}{f(T)}-p\right\| \leq \frac{\sum_{n=m}^{\infty}(n-p) a_{n}\|T\|^{n-p}}{1-\sum_{n=m}^{\infty} a_{n}\|T\|^{n-p}}
$$

Hence, (3.1) will be satisfied if:

$$
\sum_{n=m}^{\infty}\left(\frac{n-\gamma}{p-\gamma}\right) a_{n}\|T\|^{n-p} \leq 1
$$

In view of theorem 2.1, if $f \in \mathrm{AK}_{\mathrm{p}}^{\mathrm{m}}(\alpha, \beta, \delta, \mathrm{T})$ then:

$$
\sum_{n=m}^{\infty} \frac{n !(p-\beta) !\left[\frac{(n-\beta) !}{(n-\alpha-\beta) !}-\frac{(p-\beta) !}{(p-\alpha-\beta) !}+|\delta|\right]}{|\delta| p !(n-\beta) !} a_{n} \leq 1
$$
if:

By making use of (3.3) we observe that (3.2) holds true

$$
\frac{\mathrm{n}-\gamma}{\mathrm{p}-\gamma}\|\mathrm{T}\|^{\mathrm{n}-\mathrm{p}} \leq \frac{\mathrm{n} !(\mathrm{p}-\beta) !\left[\frac{(\mathrm{n}-\beta) !}{(\mathrm{n}-\alpha-\beta) !}-\frac{(\mathrm{p}-\beta) !}{(\mathrm{p}-\alpha-\beta) !}+|\delta|\right]}{|\delta| \mathrm{p} !(\mathrm{n}-\beta) !}
$$

Or equivalently:

$$
\|T\| \leq\left\{\frac{n !(p-\beta) !(p-\gamma)\left[\frac{(n-\beta) !}{(n-\alpha-\beta) !}-\frac{(p-\beta) !}{(p-\alpha-\beta) !}+|\delta|\right]}{|\delta| p !(n-\beta) !(n-\gamma)}\right\}^{\frac{1}{n-p}}
$$

This gives the desired result.

Theorem 3.2: If $\mathrm{f} \in \mathrm{AK}_{\mathrm{p}}^{\mathrm{m}}(\alpha, \beta, \delta, \mathrm{T})$ then $\mathrm{f}$ will be p-valently convex of order $\gamma\left(0 \leq \gamma<\right.$ p) in the disk $|z|<\gamma_{2}$ where:

$\gamma_{2}=\inf _{n}\left\{\frac{p n !(p-\beta) !(p-\gamma)\left[\frac{(n-\beta) !}{(n-\alpha-\beta) !}-\frac{(p-\beta) !}{(p-\alpha-\beta) !}+|\delta|\right]}{n|\delta| p !(n-\beta) !(n-\gamma)}\right\}^{\frac{1}{n-p}}$,

$(\mathrm{n} \geq \mathrm{m})$
The result is sharp for the function $\mathrm{f}$ given by (2.2).

Proof: It is enough to show that:

$$
\left\|\frac{\mathrm{Tf}^{\prime \prime}(\mathrm{T})}{\mathrm{f}^{\prime}(\mathrm{T})}+1-\mathrm{p}\right\| \leq \mathrm{p}-\gamma
$$

The result follows by application of arguments similar to the proof of theorem 3.1.

\section{RESULTS AND DISCUSSION}

\section{Convex combination}

Theorem 4.1: The class $A K_{p}^{m}(\alpha, \beta, \delta, T)$ is closed under convex combinations.

Proof: For $j=1,2, \ldots$, let $f_{j} \in A K_{p}^{m}(\alpha, \beta, \delta, T)$ where $f_{j}$ is given by:

$$
f_{j}(T)=T^{p}-\sum_{n=m}^{\infty} a_{n}, T_{j} T^{n}
$$

Then by (2.1), we obtain:

$$
\begin{aligned}
& \sum_{n=m}^{\infty} \frac{n !}{(n-\beta) !}\left[\frac{(n-\beta) !}{(n-\alpha-\beta) !}-\frac{(p-\beta) !}{(p-\alpha-\beta) !}+|\delta|\right] \\
& a_{n, j} \leq|\delta| \frac{p !}{(p-\beta) !}
\end{aligned}
$$

For $\sum_{j=1}^{\infty} \mu_{j}=1,0 \leq \mu_{j} \leq 1$, the convex combination of $\mathrm{f}_{\mathrm{j}}$ may be written as:

$$
\sum_{j=1}^{\infty} \mu_{j} f_{j}(T)=T^{p}-\sum_{n=m}^{\infty}\left(\sum_{j=1}^{\infty} \mu_{j} a_{n, j}\right) T^{n}
$$

It follows from (4.1) that:

$$
\begin{aligned}
& \sum_{n=m}^{\infty} \frac{n !}{(n-\beta) !}\left[\frac{(n-\beta) !}{(n-\alpha-\beta) !}-\frac{(p-\beta) !}{(p-\alpha-\beta) !}+|\delta|\right]\left(\sum_{j=1}^{\infty} \mu_{j} a_{n, j}\right)= \\
& \sum_{j=1}^{\infty} \mu_{j}\left(\sum_{n=m}^{\infty} \frac{n !}{(n-\beta) !}\left[\frac{(n-\beta) !}{(n-\alpha-\beta) !}-\frac{(p-\beta) !}{(p-\alpha-\beta) !}+|\delta|\right] a_{n, j}\right) \leq \\
& \sum_{j=1}^{\infty} \mu_{j}|\delta| \frac{p !}{(p-\beta) !}=|\delta| \frac{p !}{(p-\beta) !}
\end{aligned}
$$

Thus: 


$$
\sum_{j=1}^{\infty} \mu_{j} f_{j}(T) \in \mathrm{AK}_{p}^{m}(\alpha, \beta, \delta, T)
$$

Corollary 4.1: The class $\mathrm{AK}_{\mathrm{p}}^{\mathrm{m}}(\alpha, \beta, \delta, \mathrm{T})$ is a convex set. Applications of the fractional calculus

Theorem 5.1: If $\mathrm{f} \in \mathrm{AK}_{\mathrm{p}}^{\mathrm{m}}(\alpha, \beta, \delta, \mathrm{T})$ then:

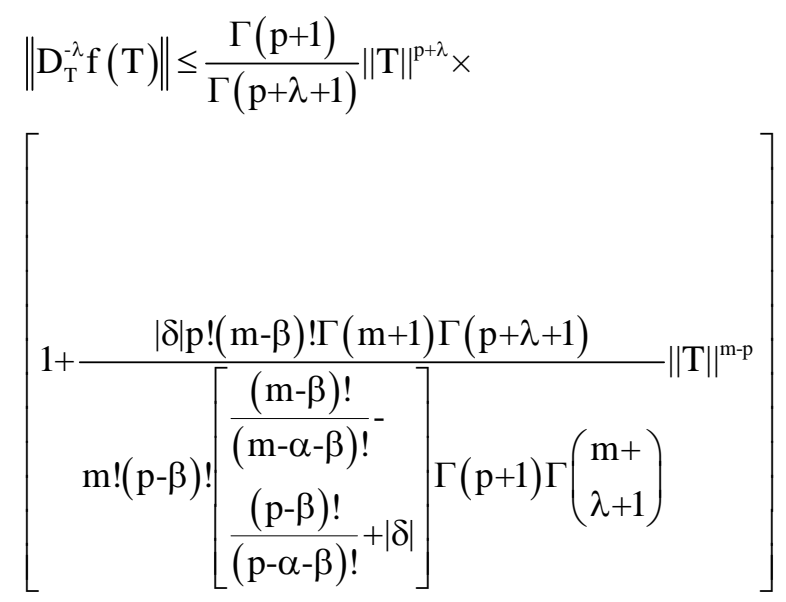

And:

$$
\begin{aligned}
& \left\|D_{T}^{-\lambda} f(T)\right\| \geq \frac{\Gamma\left(p^{+1}\right)}{\Gamma\left(p^{+\lambda+1)}\right.}\|T\|^{p+\lambda} x \\
& {\left[\begin{array}{c}
\left.1-\frac{|\delta| \mathrm{p} !(\mathrm{m}-\beta) ! \Gamma(\mathrm{m}+1) \Gamma(\mathrm{p}+\lambda+1)}{\mathrm{m} !(\mathrm{p}-\beta) !\left[\begin{array}{l}
\frac{(\mathrm{m}-\beta) !}{(\mathrm{m}-\alpha-\beta) !} \\
\frac{(\mathrm{p}-\beta) !}{(\mathrm{p}-\alpha-\beta) !}+|\delta|
\end{array}\right] \Gamma(\mathrm{p}+1) \Gamma\left(\begin{array}{l}
\mathrm{m}+ \\
\lambda+1
\end{array}\right)}\right]
\end{array}\right]}
\end{aligned}
$$

The result is sharp for the function $\mathrm{f}$ given by:

$$
f(z)=z^{p}-\frac{|\delta| p !(m-\beta) !}{m !(p-\beta) !\left[\frac{(m-\beta) !}{(m-\alpha-\beta) !}-\frac{(p-\beta) !}{(p-\alpha-\beta) !}+|\delta|\right]}
$$$$
\mathrm{z}^{\mathrm{m}},(\mathrm{p}, \mathrm{m} \in \mathrm{N})
$$

Proof: Let $\mathrm{f} \in \mathrm{AK}_{\mathrm{p}}^{\mathrm{m}}(\alpha, \beta, \delta, \mathrm{T})$. By (1.3), we deduce that:

$$
\begin{aligned}
& \frac{\Gamma\left(\mathrm{p}^{+\lambda+1)}\right.}{\Gamma(\mathrm{p}+1)} \mathrm{T}^{-\lambda} \mathrm{D}_{\mathrm{T}}^{-\lambda} \mathrm{f}(\mathrm{T})=\mathrm{T}^{\mathrm{p}}- \\
& \sum_{\mathrm{n}=\mathrm{m}}^{\infty} \frac{\Gamma(\mathrm{n}+1) \Gamma(\mathrm{p}+\lambda+1)}{\Gamma(\mathrm{p}+1) \Gamma(\mathrm{n}+\lambda+1)} \mathrm{a}_{\mathrm{n}} \mathrm{T}^{\mathrm{n}}
\end{aligned}
$$

Putting:

$$
\psi(\mathrm{n}, \lambda)=\frac{\Gamma(\mathrm{n}+1) \Gamma(\mathrm{p}+\lambda+1)}{\Gamma(\mathrm{p}+1) \Gamma(\mathrm{n}+\lambda+1)}(\mathrm{n} \geq \mathrm{m}, \mathrm{p}, \mathrm{m} \in \mathrm{N})
$$

Then, we obtain:

$$
\frac{\Gamma\left(\mathrm{p}^{+\lambda+1)}\right.}{\Gamma\left(\mathrm{p}^{+1)}\right.} \mathrm{T}^{-\lambda} \mathrm{D}_{\mathrm{T}}^{-\lambda} \mathrm{f}(\mathrm{T})=\mathrm{T}^{\mathrm{p}}-\sum_{\mathrm{n}=\mathrm{m}}^{\infty} \psi(\mathrm{n}, \lambda) \mathrm{a}_{\mathrm{n}} \mathrm{T}^{\mathrm{n}}
$$

Since, for $\mathrm{n} \geq \mathrm{m}, \psi$ is a decreasing function of $\mathrm{n}$ then we get:

$$
0<\psi(\mathrm{n}, \lambda) \leq \psi(\mathrm{m}, \lambda)=\frac{\Gamma(\mathrm{m}+1) \Gamma(\mathrm{p}+\lambda+1)}{\Gamma(\mathrm{p}+1) \Gamma(\mathrm{m}+\lambda+1)}
$$

Now, by the application of theorem 2.1 and using (5.4) we find that:

$$
\begin{aligned}
& \left\|\frac{\Gamma\left(\mathrm{p}^{+}+\lambda+1\right)}{\Gamma\left(\mathrm{p}^{+}+1\right)} \mathrm{T}^{-\lambda} \mathrm{D}_{\mathrm{T}}^{-\lambda} \mathrm{f}(\mathrm{T})\right\| \leq\|\mathrm{T}\|^{\mathrm{p}}+\sum_{\mathrm{n}=\mathrm{m}}^{\infty} \psi(\mathrm{n}, \lambda) \\
& \mathrm{a}_{\mathrm{n}}\|\mathrm{T}\|^{\mathrm{n}} \leq\|\mathrm{T}\|^{\mathrm{p}}+\psi(\mathrm{m}, \lambda)\|\mathrm{T}\|^{\mathrm{m}} \sum_{\mathrm{n}=\mathrm{m}}^{\infty} \mathrm{a}_{\mathrm{n}} \leq\|\mathrm{T}\|^{\mathrm{p}}+ \\
& \frac{|\delta| \mathrm{p} !(\mathrm{m}-\beta) ! \Gamma(\mathrm{m}+1) \Gamma(\mathrm{p}+\lambda+1)}{\mathrm{m} !(\mathrm{p}-\beta) !\left[\begin{array}{l}
\frac{(\mathrm{m}-\beta) !}{(\mathrm{m}-\alpha-\beta) !} \\
\frac{(\mathrm{p}-\beta) !}{(\mathrm{p}-\alpha-\beta) !}+|\delta|
\end{array}\right] \Gamma(\mathrm{p}+1) \Gamma(\mathrm{m}+\lambda+1)}
\end{aligned}
$$

Which gives (5.1), we also have:

$$
\begin{aligned}
& \left\|\frac{\Gamma\left(\mathrm{p}^{+\lambda+1)}\right.}{\Gamma(\mathrm{p}+1)} \mathrm{T}^{-\lambda} \mathrm{D}_{\mathrm{T}}^{-\lambda} \mathrm{f}(\mathrm{T})\right\| \geq\|\mathrm{T}\|^{\mathrm{P}}-\sum_{\mathrm{n}=\mathrm{m}}^{\infty} \psi(\mathrm{n}, \lambda) \\
& \frac{|\delta| \mathrm{p} !(\mathrm{m}-\beta) ! \Gamma(\mathrm{m}+1) \Gamma(\mathrm{p}+\lambda+1)}{\mathrm{a}_{\mathrm{n}}\|\mathrm{T}\|^{\mathrm{n}} \geq\|\mathrm{T}\|^{\mathrm{p}}-\psi(\mathrm{m}, \lambda)\|\mathrm{T}\|^{\mathrm{m}} \sum_{\mathrm{n}=\mathrm{m}}^{\infty} \mathrm{a}_{\mathrm{n}} \geq\|\mathrm{T}\|^{\mathrm{p}}-} \\
& \mathrm{m} !(\mathrm{p}-\beta) !\left[\begin{array}{c}
\frac{(\mathrm{m}-\beta) !}{(\mathrm{m}-\alpha-\beta) !} \\
\frac{(\mathrm{p}-\beta) !}{(\mathrm{p}-\alpha-\beta) !}+|\delta|
\end{array}\right] \Gamma(\mathrm{p}+1) \Gamma(\mathrm{m}+\lambda+1)
\end{aligned}
$$

Which gives (5.2). By taking $\lambda=1$ in theorem 5.1, we conclude the following corollary: 
Corollary 5.1: If $f \in f \in \mathrm{AK}_{\mathrm{p}}^{\mathrm{m}}(\alpha, \beta, \delta, \mathrm{T})$ then:

$$
\begin{aligned}
& \left\|\int_{0}^{1} \operatorname{Tf}(\mathrm{tT}) \mathrm{dt}\right\| \leq \frac{\| \mathrm{T}||^{\mathrm{p}+1}}{\mathrm{p}+1} \\
& {\left[\begin{array}{l}
\left.1+\frac{(\mathrm{p}+1)|\delta| \mathrm{p} !(\mathrm{m}-\beta) !}{(\mathrm{m}+1) \mathrm{m} !(\mathrm{p}-\beta) !\left[\frac{(\mathrm{m}-\beta) !}{(\mathrm{m}-\alpha-\beta) !}-\frac{(\mathrm{p}-\beta) !}{(\mathrm{p}-\alpha-\beta) !}+|\delta|\right]}\right] \\
\|\mathrm{T}\|^{\mathrm{m}-\mathrm{p}}
\end{array}\right]}
\end{aligned}
$$

And:

$$
\begin{aligned}
& \left\|\int_{0}^{1} \operatorname{Tf}(\mathrm{tT}) \mathrm{dt}\right\| \geq \frac{\|\mathrm{T}\|^{\mathrm{p}+1}}{\mathrm{p}+1} \\
& {\left[\begin{array}{l}
\left.1-\frac{(\mathrm{p}+1)|\delta| \mathrm{p} !(\mathrm{m}-\beta) !}{(\mathrm{m}+1) \mathrm{m} !(\mathrm{p}-\beta) !\left[\frac{(\mathrm{m}-\beta) !}{(\mathrm{m}-\alpha-\beta) !}-\frac{(\mathrm{p}-\beta) !}{(\mathrm{p}-\alpha-\beta) !}+|\delta|\right.}\right] \\
\|\mathrm{T}\|^{\mathrm{m}-\mathrm{p}}
\end{array}\right]}
\end{aligned}
$$

Proof: By definition 1.1 and theorem 5.1 for $\lambda=1$, we have $D_{T}^{-\lambda} f(T)=\int_{0}^{1} f(t T) d t$, the result is true.

Theorem 5.2: If $f \in \mathrm{AK}_{\mathrm{p}}^{\mathrm{m}}(\alpha, \beta, \delta, \mathrm{T})$ then:

$$
\left\|\mathrm{D}_{\mathrm{T}}^{\lambda} \mathrm{f}(\mathrm{T})\right\| \leq \frac{\Gamma(\mathrm{p}+1)}{\Gamma(\mathrm{p}-\lambda+1)}\|\mathrm{T}\|^{\mathrm{p}-\lambda} \times
$$

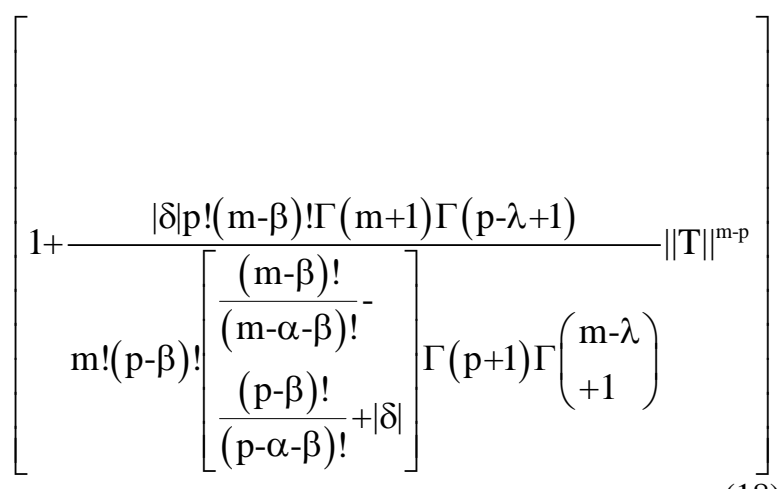

(18)

$$
\begin{aligned}
& \left\|\mathrm{D}_{\mathrm{T}}^{\lambda} \mathrm{f}(\mathrm{T})\right\| \geq \frac{\Gamma(\mathrm{p}+1)}{\Gamma(\mathrm{p}-\lambda+1)}\|\mathrm{T}\|^{\mathrm{p}-\lambda} \times \\
& :\left[\begin{array}{l}
1-\frac{|\delta| \mathrm{p} !(\mathrm{m}-\beta) ! \Gamma(\mathrm{m}+1) \Gamma(\mathrm{p}-\lambda+1)}{\mathrm{m} !(\mathrm{p}-\beta) !\left[\begin{array}{l}
(\mathrm{m}-\beta) ! \\
(\mathrm{m}-\alpha-\beta) !
\end{array}\right] \Gamma \|^{\mathrm{m}-\mathrm{p}}} \\
\frac{(\mathrm{p}-\beta) !}{(\mathrm{p}-\alpha-\beta) !}+|\delta|
\end{array}\right] \Gamma(\mathrm{p}+1) \Gamma\left(\begin{array}{l}
\mathrm{m}-\lambda \\
+1
\end{array}\right)
\end{aligned}
$$

The result is sharp for the function $\mathrm{f}$ given by (5.3).

Proof: Let $f \in \mathrm{AK}_{\mathrm{p}}^{\mathrm{m}}(\alpha, \beta, \delta, \mathrm{T})$. By (1.4), we deduce:

$$
\begin{aligned}
& \frac{\Gamma(\mathrm{p}-\lambda+1)}{\Gamma\left(\mathrm{p}^{+1}\right)} \mathrm{T}^{\lambda} \mathrm{D}_{\mathrm{T}}^{\lambda} \mathrm{f}(\mathrm{T})=\mathrm{T}^{\mathrm{p}}-\sum_{\mathrm{n}=\mathrm{m}}^{\infty} \frac{\Gamma(\mathrm{n}+1) \Gamma(\mathrm{p}-\lambda+1)}{\Gamma(\mathrm{p}+1) \Gamma(\mathrm{n}-\lambda+1)} \\
& \mathrm{a}_{\mathrm{n}} \mathrm{T}^{\mathrm{n}}=\mathrm{T}^{\mathrm{p}}-\sum_{\mathrm{n}=\mathrm{m}}^{\infty} \phi(\mathrm{n}, \lambda) \mathrm{a}_{\mathrm{n}} \mathrm{T}^{\mathrm{n}}
\end{aligned}
$$

Where:

$$
\phi(\mathrm{n}, \lambda)=\frac{\Gamma(\mathrm{n}+1) \Gamma(\mathrm{p}-\lambda+1)}{\Gamma(\mathrm{p}+1) \Gamma(\mathrm{n}-\lambda+1)}(\mathrm{n} \geq \mathrm{m}, \mathrm{p}, \mathrm{m} \in \mathrm{N})
$$

Since, for $\mathrm{n} \geq \mathrm{m}$, $\phi$ is a decreasing function of $\mathrm{n}$, thus we have:

$$
0<\phi(\mathrm{n}, \lambda) \leq \phi(\mathrm{m}, \lambda)=\frac{\Gamma(\mathrm{m}+1) \Gamma(\mathrm{p}-\lambda+1)}{\Gamma(\mathrm{p}+1) \Gamma(\mathrm{m}-\lambda+1)}
$$

Also, by using theorem 2.1,we obtain:

$$
\sum_{n=m}^{\infty} a_{n} \leq \frac{|\delta| p !(m-\beta) !}{m !(p-\beta) !\left[\frac{(m-\beta) !}{(m-\alpha-\beta) !}-\frac{(p-\beta) !}{(p-\alpha-\beta) !}+|\delta|\right]}
$$

Thus:

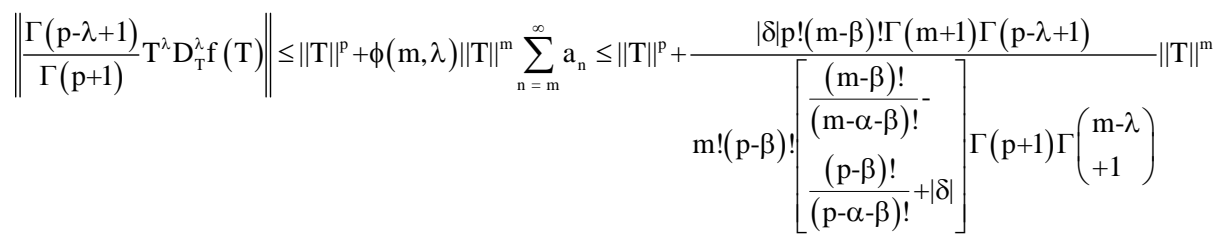


Then:

$$
\left\|\mathrm{D}_{\mathrm{T}}^{\lambda} \mathrm{f}(\mathrm{T})\right\| \leq \frac{\Gamma(\mathrm{p}+1)}{\Gamma(\mathrm{p}-\lambda+1)}\|\mathrm{T}\|^{\mathrm{p}-\lambda} \times\left[1+\frac{|\delta| \mathrm{p} !(\mathrm{m}-\beta) ! \Gamma(\mathrm{m}+1) \Gamma(\mathrm{p}-\lambda+1)}{\mathrm{m} !(\mathrm{p}-\beta) !\left[\frac{(\mathrm{m}-\beta) !}{(\mathrm{m}-\alpha-\beta) !}-\frac{(\mathrm{p}-\beta) !}{(\mathrm{p}-\alpha-\beta) !}+|\delta|\right] \Gamma(\mathrm{p}+1) \Gamma\left(\begin{array}{l}
\mathrm{m}-\lambda \\
+1
\end{array}\right)}\|\mathrm{T}\|^{\mathrm{m}-\mathrm{p}}\right]
$$

And by the same way, we conclude that:

$$
\left\|\mathrm{D}_{\mathrm{T}}^{\lambda} \mathrm{f}(\mathrm{T})\right\| \geq \frac{\Gamma(\mathrm{p}+1)}{\Gamma(\mathrm{p}-\lambda+1)}\|\mathrm{T}\|^{-\lambda-\lambda} \times\left[1-\frac{|\delta| \mathrm{p} !(\mathrm{m}-\beta) ! \Gamma(\mathrm{m}+1) \Gamma(\mathrm{p}-\lambda+1)}{\mathrm{m} !(\mathrm{p}-\beta) !\left[\frac{(\mathrm{m}-\beta) !}{(\mathrm{m}-\alpha-\beta) !}-\frac{(\mathrm{p}-\beta) !}{(\mathrm{p}-\alpha-\beta) !}+|\delta|\right] \Gamma(\mathrm{p}+1) \Gamma\left(\begin{array}{l}
\mathrm{m}-\lambda \\
+1
\end{array}\right)}\|\mathrm{T}\|^{\mathrm{m}-\mathrm{p}}\right]
$$

\section{CONCLUSION}

The operators on Hilbert space were considered recently by Xiaopei (1994), Joshi (1998), Chrakim et al. (1998), Ghanim and Darus (2008), Selvaraj et al. (2009) and Wanas and Jebur (2018).

\section{REFERENCES}

Altıntas, O., 2007. Neighborhoods of certain P-valently analytic functions with negative coefficients. Appl. Math. Comput., 187: 47-53.

Chen, M.P., H. Irmak and H.M. Srivastava, 1995. Some multivalent functions with negative coefficients defined by using a differential operator. Math. J., 6: 55-64.

Chrakim, Y., J.S. Lee and S.H. Lee, 1998. A certain subclass of analytic functions with negative coefficients for operators on Hilbert space. Math. Japonica, 47: 124-155.

Dunford, N. and J.T. Schwarz, 1988. Linear Operator, Part I, General Theory. Wiley, Hoboken, New Jersey, USA., ISBN:978-0-471-60848-6, Pages: 872.

Fan, K., 1978. Analytic functions of a proper contraction. Math. Z., 160: 275-290.

Fan, K., 1979. Julia,s lemma for operators. Math. Ann., 239: 241-245.

Ghanim, F. and M. Darus, 2008. On new subclass of analytic P-valent function with negative coefficient for operator on Hilbert space. Intl. Math. Forum, 3: 69-77.

Goodman, A.W., 1983. Univalent Functions. Vol. 1, Palygonal House Publisher, Washington, New Jersey,.

Irmak, H. and N.E. Cho, 2007. A differential operator and its applications to certain multivalently analytic functions. Hacettepe J. Math. Stat., 36: 1-6.
Joshi, S.B., 1998. On a class of analytic functions with negative coefficient for operator on Hilbert space. J. Appr. Theory Appl., 1: 107-112.

Owa, S., 1985. On certain classes of P-valent functions with negative coefficients. Simon Stevin, 59: 385-402.

Owa, S., 1992. The Quasi-Hadamard Products of Certain Analytic Functions. In: Current Topics in Analytic Function Theory, Srivastava, H.M. and S. Owa (Eds.). World Scientific Publishing Company, Singapore, Asia, pp: 234-251.

Selvaraj, C., A.J. Pamela and M. Thirucheran, 2009. On a subclass of multivalent analytic functions with negative coefficients for contraction operators on Hilbert space. Int. J. Contemp. Math. Sci., 4: 447-456.

Wanas, A.K. and A.H. Majeed, 2018. Differential subordinations for higher-order derivatives of multivalent analytic functions associated with dzioksrivastava operator. Intl. J. Anal. Appl., 16: 594-604.

Wanas, A.K. and S.K. Jebur, 2018. Geometric Properties for a family of P-valent Holomorphic Functions with negative coefficients for operator on hilbert space. J. Al-Qadisiyah Comput. Sci. Math., 10: 1-5.

Wanas, A.K., 2015. On sandwich theorems for higher-order derivatives of multivalent analytic functions associated with the generalized Noor integral operator. Asian Eur. J. Math., 8: 1-14.

Wanas, A.K., 2017. Differential subordination results defined by new class for higher-order derivatives of multivalent analytic functions. Int. J. Adv. Res. Sci. Eng. Tech., 4: 4363-4368.

Xiaopei, Y., 1994. A subclass of analytic P-valent functions for operator on Hilbert space. Math. Japonica, 40: 303-308. 